\title{
PRINCIPALES ESPECIES DE COLEÓPTEROS NECRÓFAGOS PRESENTES EN CARROÑA DE CERDOS EN LA COMARCA DE LA LITERA (HUESCA)
}

\author{
M. Castillo-Miralbés $(*)$
}

\begin{abstract}
RESUMEN
La finalidad del estudio es averiguar cuáles son los coleópteros necrófagos más frecuentes en un hábitat carroñero, utilizando cadáveres de cerdos, en la comarca de La Litera en Huesca, durante las cuatro estaciones del año y en dos ambientes distintos, al sol y a la sombra. En total se identificaron dieciocho especies pertenecientes a cinco familias de coleópteros.
\end{abstract}

Palabras clave: Coleoptera, Cleridae, Cholevidae, Dermestidae, Nitidulidae, Silphidae, necrófagos, carroña, Huesca, España.

\section{ABSTRACT \\ Main species of "Necrophagous Coleoptera" collected on pig carrion in "La Litera region" (Huesca)}

The purpose of the investigation carried out was to find out which are the "Necrophagous Coleoptera" arriving to carrion most frequently. Domestic pig carcasses were exposed in two different sites (sun and shade) during the four seasons of the year in "La Litera region", Huesca. Eighteen "Coleoptera species" were identified taxonomically belonging to five different families.

Key words: Coleoptera, Cleridae, Cholevidae, Dermestidae, Nitidulidae, Silphidae, necrophagous, carrion, Huesca, Spain.

\section{Introducción}

Se han estudiado las especies de coleópteros necrófagos asociadas a cadáveres de cerdos. El experimento se ha realizado durante las cuatro estaciones del año y en dos ambientes distintos, exponiendo los cadáveres al sol y a la sombra. Algunas especies de coleópteros aparecen frecuentemente asociadas a los cadáveres pues se alimentan de carroña o de los artrópodos que se encuentran en ese medio cadavérico. Los estadios larvarios de estos coleópteros, son necrófagos (Braack, 1987;
Catts \& Goff, 1992). También se ha estudiado la participación de estos coleópteros en el proceso de descomposición, constatando si hubo o no, distribución exclusiva de especies por zonas y estaciones, y comprobando si su acción depredadora alteró el proceso de descomposición.

Los resultados de este estudio servirán para documentar datos de las especies que puedan ser útiles a la entomología forense, disciplina que combina la entomología con las ciencias médicas legales y forenses, con el fin de esclarecer algunas de las incógnitas que generalmente rodean a los cadá-

* c/ Albalate n ${ }^{\circ}$ 13. 22510 Binaced (Huesca). 
veres encontrados en condiciones no naturales (Keh, 1985; Magaña \& Hernández, 2000). La importancia de los coleópteros necrófagos para los estudios forenses fue citada por diversos autores (Smith, 1986; Catts \& Haskell, 1990; De Souza \& Linares, 1997) y su potencial importancia en los investigaciones forenses fue mencionada por diversos autores (Goff \& Odom, 1987; Leclercq \& Verstraeten, 1988; Goff \& Flynn, 1991; Adair \& Kondratieff, 1996; Castner, 2000).

\section{Material y métodos}

El experimento se ha realizado en una finca agrícola-ganadera ubicada en el término municipal de Esplús, provincia de Huesca, a una altitud sobre el nivel del mar de $281 \mathrm{~m}$. En la misma se eligieron dos emplazamientos separados $300 \mathrm{~m}$ entre sí. El primero (exposición al sol) está situado en una zona sin vegetación y el segundo (exposición en sombra) tiene una cobertura arbórea que está compuesta en su mayor parte por álamos temblones (Populus tremula) y pseudoacacias (Gleditsia triacanthos) junto a arbustos de rubia peregrina (Rubia tinctorum) y zarzas (Rubus ulmifolius).

Como modelo animal se han utilizado 16 cerdos domésticos (Sus scrofa), 4 en cada experimento, de raza híbrida entre Landrace x Large White, de pesos comprendidos entre $8.500 \mathrm{~g}$ y $13.100 \mathrm{~g}$.

El estudio se realizó en cada una de las cuatro estaciones del año, durante los treinta días que siguieron a la muerte del animal, salvo la prueba invernal en la que el estudio se realizó durante ciento veinte días. Las fechas para cada prueba fueron las siguientes: $1^{\text {a }}$, otoño de 1997, desde el 22.09.97 hasta el 22.10.97. $2^{\mathrm{a}}$, verano de 1998, desde el 13.08.98 hasta el 02.09.98. $3^{\mathrm{a}}$, invierno de 1998-99, desde el 20.12.98 hasta el 19.04.99. $4^{\text {a }}$, primavera de 1999, desde el 23.05.99 hasta el 18.06.99.

En cada estación se colocaron cuatro cerdos, dos en cada zona, separados entre sí unos $10 \mathrm{~m}$. El muestreo se realizó diariamente y aproximadamente a la misma hora. Con el objeto de evitar el ataque de vertebrados carroñeros, cada cerdo fue introducido en una jaula metálica de $90 \times 70 \times 45$ $\mathrm{cm}$, con un paso de malla de $1,3 \mathrm{~cm}$ de lado. Para mantener siempre la ubicación original y facilitar el muestreo bajo el cadáver, cada jaula tiene cuatro patas, de $25 \mathrm{~cm}$ de largo, introducidas en tubos clavados al sustrato. Para facilitar el muestreo, en cada una de las pruebas, las jaulas fueron situadas sobre una capa de arena de río de unos $10 \mathrm{~cm}$ de espesor.
Aunque el proceso de descomposición en la naturaleza es un proceso continuo (Schoenly \& Reid, 1987), por razones prácticas se ha dividido en cuatro estados, utilizando criterios físicos y de presencia /ausencia de larvas alimentándose del cadáver. Los estados de descomposición reconocidos y la denominación de los mismos sigue la terminología empleada y recomendada en entomología forense: (1) Estado fresco: desde la muerte hasta que los gases provocados por las fermentaciones hacen que el cuerpo comience a hincharse. (2) Estado hinchado: desde que comienza la acumulación de gases hasta que la presión sobre los tejidos o el consumo por parte de los insectos necrófagos, hacen perder la estanqueidad del organismo y el cuerpo se deshincha. (3) Estado descomposición activa: Es la fase de mayor actividad de las larvas de los insectos necrófagos, consumiendo los tejidos. Comienza con la salida de los gases y finaliza cuando ya no queda recurso alimenticio para las larvas necrófagas, por lo que abandonan del cadáver para pupar en el sustrato. (4) Estado de descomposición avanzada: desde el momento en que ya no quedan larvas alimentándose, hasta que se acaba la prueba. Del cadáver ya sólo queda un estroma compacto más o menos seco formado por tejidos sin consumir y otras materias orgánicas como son los huesos y los cartílagos.

\section{Resultados}

En este estudio se han recolectado principalmente 18 especies de coleópteros de biología necrófaga, pertenecientes a 5 familias. La Tabla I muestra el listado de las especies encontradas, además se señala su presencia en el proceso de descomposición a través de los estados en que se capturaron, indicando la estación y el ambiente. Los coleópteros adultos de las familias Silphidae y Cleridae son depredadores, el resto de las familias se alimentan de carroña (Smith, 1986; Giachino, \& Tosti-Croce, 1986). Se han capturado e identificado larvas de las familias, Silphidae y Dermestidae, que son de naturaleza estrictamente necrófaga. También se han identificado larvas de la familia Cholevidae, su presencia en el medio cadavérico esta justificada puesto que son necrófagas facultativas. La Tabla II muestra a partir de que día de exposición se capturaron esas larvas de cada familia, para cada estación y ambiente. Todas las especies de coleópteros citados en este estudio, ya estaban citadas en España y representan el 6,59 \% del total de artrópodos identificados en el experimento (CastilloMiralbés, 2000). 
Tabla I.- Especies de coleópteros necrófagos presentes en carroña de cerdos, en cada uno de los estados de descomposición para cada ambiente y estación.

Table I. - Species of necrophagous coleoptera collected on pig carcass in each one of the states of decomposition for every ambiance and station.

\begin{tabular}{|c|c|c|c|c|c|}
\hline Familia & Especie & Fresco & Hinchado & Activa & Avanzada \\
\hline \multirow{7}{*}{ Cleridae } & \multirow{3}{*}{ Necrobia rufficolis (Fabricius, 1775) } & \multirow{3}{*}{-} & \multirow{3}{*}{$\begin{array}{l}\mathrm{P} / \text { sol y } \\
\text { sombra }\end{array}$} & $0,1 / \mathrm{sol}$ & \multirow{3}{*}{$\mathrm{V}, \mathrm{P} /$ sombra } \\
\hline & & & & V/sombra & \\
\hline & & & & $\begin{array}{l}\mathrm{P} / \text { sol y } \\
\text { sombra }\end{array}$ & \\
\hline & \multirow[b]{2}{*}{ Necrobia rufipes (DeGeer, 1775) } & \multirow[b]{2}{*}{-} & $\mathrm{O}, \mathrm{V} / \mathrm{sol}$ & \multirow{2}{*}{$\begin{array}{l}\mathrm{O}, \mathrm{V}, \mathrm{I}, \mathrm{P} / \\
\text { sol y } \\
\text { sombra }\end{array}$} & \multirow{2}{*}{$\begin{array}{c}\mathrm{O}, \mathrm{V}, \mathrm{I}, \mathrm{P} / \mathrm{sol} \\
\text { y sombra }\end{array}$} \\
\hline & & & $\begin{array}{c}\text { I, P/sol y } \\
\text { sombra }\end{array}$ & & \\
\hline & \multirow{2}{*}{ Necrobia violacea (Linnaeus, 1758) } & \multirow[t]{2}{*}{ I/ sombra } & $\begin{array}{l}\text { I/ sol y } \\
\text { sombra }\end{array}$ & \multirow{2}{*}{$\begin{array}{l}\text { I. P / sol y } \\
\text { sombra }\end{array}$} & \multirow{2}{*}{$\begin{array}{l}\text { I/ sol y } \\
\text { sombra }\end{array}$} \\
\hline & & & $\mathrm{P} /$ sombra & & \\
\hline \multirow{5}{*}{ Cholevidae } & \multirow[b]{2}{*}{ Catops coracinus (Kellner, 1846) } & \multirow[b]{2}{*}{ I/ sombra } & \multirow[b]{2}{*}{$\begin{array}{c}\text { V, I/ } \\
\text { sombra }\end{array}$} & V/sombra & O/sombra \\
\hline & & & & $\begin{array}{l}\text { I/sol y } \\
\text { sombra }\end{array}$ & $\begin{array}{l}\text { I/sol y } \\
\text { sombra }\end{array}$ \\
\hline & \multirow[b]{2}{*}{ Catops fuscus (Panzer, 1794) } & \multirow[b]{2}{*}{ I/ sombra } & \multirow[b]{2}{*}{$\begin{array}{c}\mathrm{V}, \mathrm{II} \\
\text { sombra }\end{array}$} & V/sombra & O/sombra \\
\hline & & & & $\begin{array}{l}\text { I/ sol y } \\
\text { sombra }\end{array}$ & $\begin{array}{l}\text { I/ sol y } \\
\text { sombra }\end{array}$ \\
\hline & Ptomaphagus tenuicornis (Rosenhauer, 1856) & - & $\mathrm{V} /$ sombra & V/sombra & - \\
\hline \multirow{4}{*}{ Dermestidae } & \multirow[b]{2}{*}{ Dermestes frischii (Kugelann, 1792) } & \multirow[b]{2}{*}{$\mathrm{P} / \mathrm{sol}$} & $\mathrm{O} / \mathrm{sol}$ & $\mathrm{O} / \mathrm{sol}$ & $\mathrm{O} / \mathrm{sol}$ \\
\hline & & & $\begin{array}{l}\text { I, P/sol y } \\
\text { sombra }\end{array}$ & $\begin{array}{l}\mathrm{V}, \mathrm{I}, \mathrm{P} / \mathrm{sol} \\
\text { y sombra }\end{array}$ & $\begin{array}{c}\mathrm{V}, \mathrm{I}, \mathrm{P} / \mathrm{sol} \mathrm{y} \\
\text { sombra }\end{array}$ \\
\hline & \multirow[b]{2}{*}{ Dermestes undulatus (Brahm, 1790) } & \multirow[b]{2}{*}{$\begin{array}{l}\mathrm{P} / \text { sol y } \\
\text { sombra }\end{array}$} & I/sol & \multirow[b]{2}{*}{$\begin{array}{l}\text { I, P / sol y } \\
\text { sombra }\end{array}$} & $\mathrm{V} / \mathrm{sol}$ \\
\hline & & & $\begin{array}{l}\mathrm{P} / \text { sol y } \\
\text { sombra }\end{array}$ & & $\begin{array}{l}\text { I, P / sol y } \\
\text { sombra }\end{array}$ \\
\hline \multirow{6}{*}{ Nitudilidae } & Carpophilus freemani ( Dobson, 1956) & - & $\mathrm{P} / \mathrm{sol}$ & $\begin{array}{l}\mathrm{P} / \text { sol y } \\
\text { sombra }\end{array}$ & $\mathrm{P} /$ sombra \\
\hline & Melighetes sp. & - & I/ sol & - & - \\
\hline & Nitidula bipunctata (Linnaeus, 1758) & - & I/sombra & $\begin{array}{l}\text { I/ sol y } \\
\text { sombra }\end{array}$ & $\mathrm{I} / \mathrm{sol}$ \\
\hline & Nitidula carnaria $\quad$ (Schaller, 1783) & I/sombra & I/sombra & $\begin{array}{l}\text { I/ sol y } \\
\text { sombra }\end{array}$ & $\begin{array}{l}\text { I/ sol y } \\
\text { sombra }\end{array}$ \\
\hline & Nitidula flavomaculata (Rossi, 1790) & $\begin{array}{l}\text { I/ sol y } \\
\text { sombra }\end{array}$ & $\begin{array}{c}\text { I/sol } \\
\mathrm{P} / \text { sombra }\end{array}$ & $\begin{array}{c}\text { O, V/sol y } \\
\text { sombra } \\
P / \text { sol }\end{array}$ & $\begin{array}{c}\text { O, V / sol y } \\
\text { sombra } \\
\mathrm{P} / \text { sol }\end{array}$ \\
\hline & Soronia punctatissima (Illiger, 1794) & - & $\mathrm{P} /$ sombra & $\mathrm{P} /$ sombra & - \\
\hline \multirow{6}{*}{ Silphidae } & Necrodes litoralis (Linnaeus, 1761) & - & - & O/sombra & - \\
\hline & \multirow[t]{2}{*}{ Thanatophilus sinuatus (Fabricius, 1775) } & \multirow[b]{2}{*}{$\mathrm{O} /$ sombra } & $\begin{array}{c}\text { O, VI } \\
\text { sombra }\end{array}$ & V/sombra & I, P / sombra \\
\hline & & & $\begin{array}{l}\text { I, P/sol y } \\
\text { sombra }\end{array}$ & $\begin{array}{l}\mathrm{O}, \mathrm{I}, \mathrm{P} / \mathrm{sol} \\
\text { y sombra }\end{array}$ & $\begin{array}{l}\text { O / sol y } \\
\text { sombra }\end{array}$ \\
\hline & \multirow[b]{2}{*}{ Thanatophilus ruficornis (Küster, 1851) } & \multirow[b]{2}{*}{ I/ sombra } & $\mathrm{P} /$ sombra & $\mathrm{P} /$ sombra & \\
\hline & & & $\begin{array}{l}\text { I/ sol y } \\
\text { sombra }\end{array}$ & $\begin{array}{l}\text { I/ sol y } \\
\text { sombra }\end{array}$ & I, P / sombra \\
\hline & Silpha tristis (Illiger, 1798) & - & O/sombra & $\mathrm{O} /$ sombra & - \\
\hline
\end{tabular}

O: otoño, I: invierno, V: verano, P: primavera .O: autumn , I: winter, V: summer, P: spring

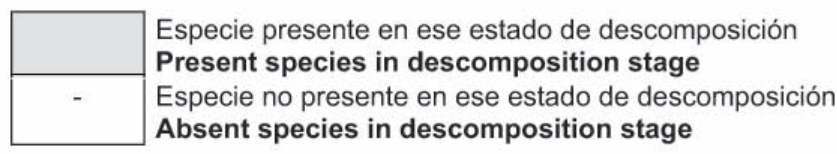


Tabla II.- Primer día de captura, de las larvas identificadas de coleópteros necrófagos, para cada estación y ambiente.

Table II.- First day of capture, of the identified larvae of necrophagous coleoptera, for every station and ambiance.

\begin{tabular}{|c|r|c|c|c|}
\hline Estación & \multicolumn{1}{|c|}{ Ambiente } & SILPHIDAE & DERMESTIDAE & CHOLEVIDAE \\
\hline \multirow{3}{*}{ OTOÑO } & sol & Día 12 & Día 20 & Día 10 \\
\cline { 2 - 5 } & sombra & Día 10 & Ausencia & Ausencia \\
\hline \multirow{3}{*}{ VERANO } & sol & Ausencia & Día 7 & Ausencia \\
\cline { 2 - 5 } INVIERNO & sombra & Ausencia & Día 10 & Ausencia \\
\cline { 2 - 5 } & sol & Ausencia & Día 101 & DÍA 81 \\
\hline \multirow{2}{*}{ PRIMAVERA } & somála 107 & Día 109 & Día 79 \\
\cline { 2 - 5 } & som & Día 18 & Día 10 & Ausencia \\
\hline
\end{tabular}

De la familia Cleridae, la especie que se capturó mayoritariamente en todos los ambientes y estaciones fue Necrobia rufipes (DeGeer, 1775). De la familia Cholevidae, se recolectaron ejemplares en todas las estaciones, salvo en la primaveral. Con respecto a las especies de la familia Dermestidae, los ejemplares de la especie Dermestes frischii (Kugelan,1792) fueron los que se capturaron mayoritariamente. De la familia Nitidulidae, la especie Nitidula flavomaculata (Rossi, 1790) fue la más recolectada y la única que se capturó en todas las estaciones y ambientes. $Y$ de la última familia estudiada, la Siphidae, dos especies fueron las más recolectadas, Thanatophilus sinuatus (Fabricius, 1775) en las pruebas de otoño y primavera y Thanatophilus ruficornis (Küster, 1851) en la invernal. Llama la atención la ausencia de capturas de coleópteros de la familia Silphidae durante la prueba veraniega, en la zona soleada.

En este estudio no se ha encontrado ninguna especie de coleópteros necrófagos, que por ser endémica o con distribución geográfica limitada, pudiera ser utilizada para detectar posibles movimientos post-mortem en las investigaciones forenses. Y en ninguna prueba, se comprobó que la actividad depredadora de los coleópteros, ni la necrófaga de sus larvas, alterara el proceso de descomposición.

\section{Discusión}

Se han recolectado coleópteros necrófagos en todos los estados de descomposición, aunque estudiándolos independientemente por familias, se encontraron distribuciones diferentes. También ha sido diferente la acción llevada acabo por cada familia en ese ecosistema. Los ejemplares de la familia Dermestidae fueron los que más presente estuvieron y los que más actividad realizaron. Su presencia en el medio cadavérico es retrasada por algunos autores (Payne, 1965; Leclercq, 1978; Beranger, 1990), hasta estados cercanos a la sequedad de todos los tejidos. En este estudio se han capturado tempranamente, coincidiendo con los trabajos de otros autores (Tantawi et al., 1996; Avila \& Goff, 1998). Sus larvas fueron las principales consumidoras, en todas las pruebas, de los restos de piel y estroma seco de los tejidos que quedaban en el estado de descomposición avanzada. Esta actividad consumidora, ya fue comprobada y destacada en diversos trabajos (Lord \& Burger, 1984; Early \& Goff, 1986; Richards \& Goff, 1997).

De la familia Siphidae se han recolectado ejemplares adultos desde el inicio de la descomposición, en los estados fresco e hinchado. Esta presencia temprana se había constatado en trabajos como los de Reed (1958) y Shean et al., (1993), además muy pronto se recolectaron larvas de esta familia. Concretamente al octavo día de la prueba primaveral y al día décimo de exposición en la prueba otonal. Su importancia como consumidores del recurso ha sido citada por Nabaglo (1973).

Se han capturado ejemplares de la familia Cleridae desde los primeros estados de descomposición, estados en que fueron citados por diversos autores (Rodriguez \& Bass, 1983; Byrd \& Castner, 2000) . En este estudio no se encontraron larvas de esta familia, mientras que Anderson et al., (1996), las recolectaron a partir del día noventa y cuatro de exposición. En nuestro caso todos los trabajos acabaron a los treinta días, salvo la prueba invernal y en esta tampoco se identificaron. 
Los ejemplares de la familia Cholevidae se han capturado en mayor cantidad en la zona de sombra, Ketner \& Street, (1990), encontraron más abundantes los ejemplares de esta familia en la zona boscosa. En nuestro caso se recolectaron en los inicios del proceso de descomposición salvo en la prueba otoñal, coincidiendo con el trabajo de Bourel et al., (1999), donde fueron capturados a partir del quinto día de exposición.

De los resultados obtenidos y de su comparación con los de otros autores, se concluye que son necesarios estudios concretos para cada zona o región climática, para conocer a fondo la entomofauna carroñera si queremos aplicar esos conocimientos de entomología, a las ciencias forenses.

\section{AGRADECIMIENTOS}

Quiero dejar constancia de mi agradecimiento a los siguientes entomólogos especialistas por su ayuda en la identificación de los ejemplares colectados. A la Dra. Marina Blas del Dpto. de Biologia Animal (Universitat de Barcelona) por la identificación de los ejemplares de la familias Cholevidae y Colonidae; al Dr. Pablo Bahillo de la Puebla por su ayuda en la identificación de las especies de la familia Cleridae; al Dr. Javier Pérez Valcarcel de A Coruña por la determinación de las especies de la familia Silphidae. Al Dr. Michael Geisthardt del Museum Wiesbaden (Wiesbaden, Alemania) por la identificación de ejemplares de la familia Dermestidae. Y al Dr. José Carlos Otero Gonzalez del Dept. de Biología (Universidad de Santiago de Compostela, A Coruña) por la identificación de ejemplares de la familia Nitidulidae.

\section{Referencias}

Adair, T.W. \& Kondratief, B.C., 1996. The ocurrence of Nitidula flavomaculata (Coleoptera; Nitidulidae) on a human corpse. Entomological News, 107:233236.

Anderson, G.S. \& Vanlaerhoven, S.L., 1996. Initial studies on insect succession on carrion in Southwestern Britsh Columbia. Journal of Forensic Sciences, 41(4): 617-625.

Avila, F.W. \& Goff, M.L., 1998. Arthropod succession patterns onto burnt carrion in two contrasting habitats in the Hawaiian Islands. Journal of Forensic Sciences, 43(3):581-586.

Beranger, L.P., 1990. Les insectes dans lénquête policière. Boubée, Paris. 56 pp.

Bourel, B., Martin-Bouyer, L., Hédouin, V, Cailliez, J.C., Derout, D. \& Gosset, D., 1999. Necrophilus insect succession on rabbbit carrion in sand dune habitats in Northern France. Journal of Medical Entomology. 36(4): 420-425.
BRAACK, L., 1987. Community dynamics of carrionattendant arthropods in tropical african woodland. Oecologia, 72: 402-409.

Byrd, J.H. \& CASTNER, J.L., 2000. Insects of forensic importance. In: J.H. Byrd \& J.L. Castner. Forensic Entomology: the utility of arthropods in legal investigations. CRC Press, Florida: 43-80.

CAstillo-Miralbés, M., 2000. Estudio de la entomofauna asociada a los cadáveres en la Región Altoaragonesa. Universidad de Zaragoza. Tesis doctoral inédita. $318 \mathrm{pp}$.

CASTner, J.L., 2000. General entomology and artrhopod biology. In: J.H. Byrd \& J.L. Castner. Forensic Entomology: the utility of arthropods in legal investigations. CRC Press, Florida: 17-42.

CATTS, E.P. \& GofF, M.L., 1992. Forensic entomology in criminal investigations. Annual Reviews of Entomology, 37: 253-272.

CAtTs, E.P. \& Haskell, N.H., 1997. Entomology and death: a procedural guide. Joyce's Print Shop, Inc., Clemson. $182 \mathrm{pp}$.

De Souza, A.M. \& Linhares, A.X., 1997. Diptera and Coleoptera of potential forensic importance in southeastern Brazil: relative abundance and seasonality. Medical and Veterinary Entomology, 11(1): 8-12.

EARly, M. \& GofF, M.L., 1986. Arthropod succession patterns in exposed carrion on the Island of O'Ahu, Hawaiian Islands, USA. Journal of Medical Entomology, 23(5): 520-531.

Giachino, P.M. \& Tosti-Croce, E., 1986. Note su alcune specie di Catopidi (Coleoptera, Catopidae) associate alla decomposizione di un cadávere di micromammifero. Bulletin del Museo Regionale di Scienze naturale Torino, 4(2): 395-411.

Goff, M.L. \& Odom, C.B., 1987. Forensic entomology in the Hawaiian Islands. Three case studies. The American Journal of Forensic Medecine and Pathology, 8(1): 45-50.

$\mathrm{KEH}$, B., 1985. Scope and applications of forensic entomology. Annual Review of Entomology, 30: 137-154.

Kentner, E. \& Streit, B., 1990. Temporal distribution and habitat preference of congeneric insect species found at rat carrion. Pedobiologia, 34:347-359.

LECLERCQ, M., 1978. Entomologie et médecine légale. Datation de la mort. Masson. Paris. 100 pp.

LeClercQ, M. \& Verstraeten, C., 1988. Entomologie et médecine légale.Datation de la mort: insectes et autres arthropodes trouvés sur les cadavres humains. Bulletin Annuel de la Société Royal Belge Entomologique, 124: 311-317.

LORD, W.D. \& Burger, J.F., 1984. Arthropods Associated with Herring Gull (Larus argentatus) and Great Black-backed Gull (Larus marinus) Carrion on Islands in the Gulf of Maine. Environmental Entomology, 13:1261-1268. 
Magaña, C. \& Hernández, M., 2000. Aplicaciones de la entomología forense en las investigaciones médico-legales. IX Congreso Ibérico de Entomología. Zaragoza 4-8 de Julio 2000.

NABAGLO, L., 1973. Participation of invertebrates in descomposition of rodent carcasses in forest ecosystems. Ekologia Polska, 21(18): 251-270.

PAYNE, J.A., 1965. A summer carrion study of the baby pig Sus scrofa Linnaeus. Ecology, 46(5): 592-602.

ReED, H.B., 1958. A study of dog carcass communities in Tennessee, whith special reference to the insects. The American Midland Naturalist, 59(1): 213-245.

RichaRdS, E.N. \& GoFf, M. L., 1997. Arthropod succession on exposed carrion in three contrasting tropical habitats on Hawaii Island, Hawaii. Journal of Medical Entomology, 34(3): 328-339.

RodRIGUEZ, W.C. \& BASS, W. M., 1983. Insect activity and its relationship to decay rates of human cadávers in East Tennessee. Journal of forensic Sciences, 28(2): 423-432.

Schoenty, K. \& ReID,W., 1987. Dynamics of heterotrophic succession in carrion arthropd assemblages: discrete seres or a continuum of change? Oecologia, 73: 192-202.
Shean, B.S., Messinger, L. \& Papworth, M., 1993. Observations of differential decomposition on sun exposed v. shaded pig carrion in coastal Washington state. Journal of Forensic Sciences, 38(4):938-949.

SмIтH, K.G.V., 1986. A manual of forensic entomology. British Museum Publications. London. 205 pp.

TANTAWI, T.I., Kady-Em-El, GreEnBerg, B. \& GHAFfarHA-EL, 1996. Arthropod succession on exposed rabbit carrion in Alexandria, Egypt. Journal Medical Entomology, 33(4): 566-580. 